\title{
Early Secreted Antigen ESAT-6 of Mycobacterium Tuberculosis Promotes Apoptosis of Macrophages via Targeting the MicroRNA155-SOCS1 Interaction
}

\author{
Shaojun Yang Fake Li Shuangrong Jia Kejun Zhang Wenbing Jiang Ya Shang \\ Kai Chang Shaoli Deng Ming Chen
}

Department of Clinical Laboratory, Institute of Surgery Research, Daping Hospital, Third Military Medical University, Chongqing, China

\author{
Keywords \\ Apoptosis $・$ ESAT- $6 \cdot$ microRNA-155 $\bullet$ SOCS1 $・$ TLR2
}

\begin{abstract}
Background: The early secreted antigenic target 6-kDa protein (ESAT-6) of Mycobacterium tuberculosis (Mtb) not only acts as a key player for virulence but also exhibits a strong immunotherapeutic potential against Mtb. However, little is known about the molecular basis for its potential in immunotherapy. The present study was designed to unravel the role of miRNA-155 in ESAT-6-mediated enhancement of host immunity and apoptosis in macrophages. Methods: Lentivirus-mediated miR-155 sponge and miR-155 and SOCS1 overexpression vectors were developed in macrophages. TLR2- or p65-specific siRNA knockdown was employed to silence TLR2 or p65. Quantitative polymerase chain reaction and western blotting analyses were performed to determine mRNA and protein expression levels, respectively. Macrophage apoptosis was analyzed by flow cytometry. Results: ESAT- 6 significantly increased miR-155 expression, which was dependent on TLR2/NF-KB activation in macrophages. Induced expression of miRNA-155 was required for the ESAT-6-mediated protective immune response and macrophage apoptosis. ESAT-6 promoted macrophage apoptosis by targeting the miR-155-SOCS1 pathway. The differential expression levels of TLR2, $\mathrm{BIC}$, and SOCS1 were involved in regulating the immune response in human peripheral blood mononuclear cells of patients with active tuberculosis (TB) and latent TB (LTB). Conclusion: ESAT-6 promotes apoptosis of macrophages via targeting the miRNA155-SOCS1 interaction.
\end{abstract}




\section{Introduction}

Tuberculosis (TB) continues to be a global health issue. According to the World Health Organization Global Tuberculosis Report 2013, an estimated 8.6 million people developed TB and 1.3 million died from the disease in 2012 [1]. The pathogen responsible for this widespread and persistent infection is Mycobacterium tuberculosis (Mtb), which often resides in macrophages and eventually induces the death of its host cells. The survival of $M t b$ is dependent on its capacity to block the immune response of the host. As the principal reservoir of $M t b$, macrophages also serve as the main effector cells responsible for the control of mycobacterial replication. Importantly, apoptosis is essential for macrophages to kill $M t b$, while virulent $M t b$ inhibits apoptosis but induces necrosis, which facilitates bacterial escape into the surrounding tissues to start a new cycle of infection, ultimately resulting in bacterial dissemination [2-4].

The early secreted antigenic target 6-kDa protein (ESAT-6) is one of the key antigens expressed by $M t b$, but not Bacillus Calmette-Guerin (BCG), and has been studied extensively for its potent immunomodulatory activities as a subunit vaccine [5, 6]. A recombinant BCG that contains region of difference 1 (RD1) and exports ESAT- 6 shows enhanced protection against TB [7]. The ESAT-6-induced apoptosis in macrophages was first demonstrated by Derrick et al. in 2007 [8]. Furthermore, ESAT-6 drives Th17 cell differentiation [9] and exhibits an immunotherapeutic potential against experimental TB [10]. However, the mechanism by which the ESAT-6 system-1 (ESX1)-encoded ESAT-6 induces protective immune responses and apoptosis against TB remains elusive.

MicroRNAs (miRNAs) are a class of evolutionarily conserved, single-stranded, noncoding RNAs of 20 to 22 nucleotides that control gene expression by translational repression and/ or induction of mRNA degradation. In recent years, microRNA-155 (miR-155, encoded by a noncoding gene known as BIC) has emerged as a crucial regulator of innate or adaptive immune responses. Recent studies have indicated that miR-155 has a novel role in Th1 or Th17 differentiation during microbial infections [11] and that augmented expression of miR-155 is observed in lipopolysaccharide (LPS)-induced inflammatory response [12]. Furthermore, miR-155 affects the expression of dendritic cell-specific intercellular adhesion molecule 3 grabbing non-integrin (DC-SIGN) by downregulation of PU.1 (a spleen focus forming virus proviral integration oncogene) [13].

The suppressor of cytokine signaling (SOCS) family, also known as cytokine-inducible SH2-containing proteins and STAT-induced STAT inhibitor, consists of eight members (SOCS1-7 and CISH), with SOCS1 being the most characterized, each of which shares a central modulator with a SH2 domain and a C-terminal SOCS box [14]. They are key regulators of the cross-regulation of cytokine secretion by targeting the JAK/STAT pathway in different immune cells. SOCS1 is a well-established key negative regulator of the JAK/STAT pathway and inhibits proinflammatory cytokines such as tumor necrosis factor alpha (TNF- $\alpha$ ), interleukin-6 (IL-6), and interferon gamma (IFN- $\gamma$ ) [15]. More recently, miR-155 has been implicated as an important regulator of breast cancer and LPS-induced inflammatory response through negatively regulating SOCS1 [16].

In light of the aforementioned reports, the present study was designed to unravel the relationship between ESAT- 6 and miR-155 expression and the role of miR-155 in ESAT-6-mediated enhancement of host immunity and apoptosis of macrophages. We investigated whether ESAT-6 regulates the expression of miR-155 in macrophages; whether enhanced expression of mir-155 or knockdown of miR-155 affects SOCS1 expression, the production of cytokines such as IL-6, and apoptosis in macrophages; and whether the expression of miR-155 is dependent on Toll-like receptor 2 (TLR2)/nuclear factor kappalight-chain-enhancer of activated B cell (NF- $\kappa \mathrm{B})$ activation. We further analyzed the expression of TLR2 and BIC in LTB patients and its role in the immune responses against $M t b$, and the relationship between the expression of SOCS1 and the suppression of immune activation of the host and the survival of $M t b$ in TB patients. We believe that these studies will help demonstrate the role of the TLR2/miR-155/SOCS1 signaling axis in the immune 
response and apoptosis of macrophages as well as the novel regulatory mechanism of ESAT6 in host innate immunity against mycobacterial infections.

\section{Materials and Methods}

\section{Reagents and antibodies}

Cell culture media and fetal bovine serum (FBS) were obtained from Gibco-Invitrogen (Carlsbad, CA, USA). Cell culture antibiotics were purchased from Sigma-Aldrich (St. Louis, MO, USA). Antibodies against caspase-3 and p65 were purchased from Cell Signaling Technology (Danvers, MA, USA), and antibodies against TLR2, SOCS1, and $\beta$-actin were purchased from Abcam (Cambridge, MA, USA). Recombinant ESAT-6 protein $(1 \mathrm{mg} / \mathrm{ml}$ ) was purchased from Kitgen Bio-tech (Hangzhou, China). All other chemicals and reagents used in the present study were purchased from Sigma-Aldrich.

\section{Cell culture and treatment}

The murine macrophage cell line RAW264.7 was obtained from the American Type Culture Collection (TIB-71). Mtb and M. bovis BCG strain were provided by the Chinese Center for Disease Control and Prevention and were grown in Middlebrook 7H9 as described previously [17]. The RAW264.7 cells were cultured in Dulbecco's modified Eagle's medium (DMEM) supplemented with 10\% FBS and 100 U/ $\mathrm{ml}$ penicillin/streptomycin at $37^{\circ} \mathrm{C}$ in a humidified atmosphere with $5 \% \mathrm{CO}_{2}$. Macrophage cultures were treated with $5 \mu \mathrm{g} / \mathrm{ml}$ ESAT-6 as described previously [17] for 0-24 h; total RNAs and lysates were then prepared for various assays.

\section{Cell transfection}

Cell transfection was performed using Lipofectamine 2000 (Santa Cruz Inc., Santa Cruz, CA, USA) according to the manufacturer's instructions. RAW264.7 macrophages were transfected with 50 nM TLR2specific small interfering RNA (Santa Cruz Inc.) to silence TLR2 or were transfected with $50 \mathrm{nM}$ p65-specific small interfering RNA (Santa Cruz Inc.) to knock down p65.

\section{Lentivirus preparation and in vitro infection}

Lentiviral vectors expressing miR-155 sponge, miR-155, or SOCS1 were generated by GeneChem (Shanghai, China). Cell infections were performed in triplicate according to the manufacturer's recommendations, with a multiplicity of infection (MOI) of approximately twenty.

Subjects

Patients recruited into the current study were seen at the Department of Clinical Laboratory at Daping Hospital, Third Military Medical University (Chongqing, China), between March 2013 and April 2014. All patients had received the T-Spot® TB test. Patients who were pregnant, immunosuppressed, or had an autoimmune disease were excluded from this study.

The patients were divided into three groups according to their TB status: TB-negative $(n=20)$, active TB ( $\mathrm{n}=20)$, and latent TB (LTB; $\mathrm{n}=20$ ) [18-20]. The TB-negative group included subjects who tested negative with the T-Spot® TB test. The active TB group included subjects who either had $M t b$ cultures grown from a clinical specimen or a positive T-Spot ${ }^{\circ}$ TB test, and were reported to the Chinese Center for Disease Control and Prevention. The LTB group included subjects with either a positive tuberculin skin test, a positive T-Spot $\AA$ TB test, or both, but with no signs or symptoms of active TB disease and no positive cultures for $M t b$. Moreover, radiological and clinical examinations were also performed on each of the subjects to confirm active TB in pulmonary TB patients and to exclude individuals with LTB disease. Each of the subjects provided a written informed consent, and the study protocol was reviewed and approved by the Institutional Bioethics Committee of the Third Military Medical University.

Quantitative PCR ( $q P C R$ ) analysis

Total RNAs were extracted from RAW264.7 cells treated with EAST-6 ( $5 \mu \mathrm{g} / \mathrm{ml})$ or peripheral blood mononuclear cells (PBMCs) using TRIzol reagent (Gibco-Invitrogen, Carlsbad, CA, USA). The real-time quantitative reverse transcription polymerase chain reaction (RT-PCR) for miR-155 was performed with 
Table 1. Primers for qRT-PCR

\begin{tabular}{ll}
\hline Primers & Sequences $5^{\prime} \rightarrow 3^{\prime}$ \\
\hline Mouse BIC-F & CTTGCTGAAGGCTGTATGCT \\
Mouse BIC-R & ATTCTCGACTGACATCTAGG \\
Mouse SOCS1-F & CTCCGTGACTACCTGAGTTCCTT \\
Mouse SOCS1-R & ATGAGGTCTCCAGCCAGAAGTG \\
Mouse IL6-F & GACTTCCATCCAGTTGCCTTCTT \\
Mouse IL6-R & TGTAATTAAGCCTCCGACTTGTGAA \\
Mouse TNF $\alpha-F$ & AAGACACCATGAGCACAGAAAGC \\
Mouse TNF $\alpha-\mathrm{R}$ & GCCACAAGCAGGAATGAGAAGAG \\
Mouse IFN $\gamma-\mathrm{F}$ & GAGGTCAACAACCCACAGGT \\
Mouse IFN $\gamma-\mathrm{R}$ & GGGACAATCTCTTCCCCACC \\
Mouse iNOS-F & AACTACTGCTGGTGGTGACAAG \\
Mouse iNOS-R & TGAAGGTGTGGTTGAGTTCTCTAAG \\
Mouse $\beta-A C T I N-F$ & GCCTCACTGTCCACCTTCCA \\
Mouse $\beta-A C T I N-R$ & AGCCATGCCAATGTTGTCTCTTAT \\
Human BIC-F & CTCTAATGGTGGCACAAA \\
Human BIC-R & TGATAAAAACAAACATGGGCTTGAC \\
Human SOCS1-F & TTTTTCGCCCTTAGCGTGA \\
Human SOCS1-R & AGCAGCTCGAAGAGGCAGTC \\
Human TLR2-F & GGAGTTCTCCCAGTGTTTGGT \\
Human TLR2-R & GCAGTGAAAGAGCAATGGGC \\
Human GAPDH-F & GGAGCGAGATCCCTCCAAAAT \\
Human GAPDH-R & GGCTGTTGTCATACTTCTCATGG \\
\hline
\end{tabular}

the TaqMan microRNA Assay Kit (Applied Biosystems, Foster City, CA, USA) using specific qRT Primer Sets following the manufacturer's instructions (Applied Biosystems). U6 snRNA was used for normalization. cDNA was synthesized using a Transcriptor First Strand cDNA Synthesis Kit (Roche Diagnostics GmbH, Mannheim, Germany). The PCR was performed using a Bio-Rad CFX96 real-time thermal cycler and FastStart Essential DNA Green Master (Roche Diagnostics GmbH, Mannheim, Germany) for analyses of the expressions of BIC, TLR2, SOCS1, IL-6, IFN- $\gamma$, inducible nitric oxide synthase (iNOS), and TNF- $\alpha$, respectively. The relative expression levels of the mRNAs were normalized to that of the internal control $\beta$-actin or GAPDH using the $2^{-\Delta \Delta C t}$ cycle threshold method. All the PCR primers used in this study are listed in Table 1.

Analysis of cellular apoptosis

RAW264.7 cells were treated with $5 \mu \mathrm{g} / \mathrm{ml}$ of ESAT-6 protein, harvested at various times ( 0 to $24 \mathrm{~h}$ ), and then labeled with annexin V-FITC and propidium iodide following the manufacturer's instructions (BD Bioscience, Franklin Lakes, NJ, USA). The cells were sorted by fluorescence-activated cell sorting, and the data were analyzed using FlowJo software (Tree Star, Ashland, OR, USA) as described previously [21].

\section{Western blot analysis}

The treated cells were washed with cold phosphate-buffered saline (PBS) and were lysed in RIPA lysis buffer (Beyotime Biotechnology, Shanghai, China). After protein quantification, equal amounts of protein lysates ( $50 \mu \mathrm{g}$ each) were separated by $15 \%$ sodium dodecyl sulfate-polyacrylamide gel electrophoresis and transferred to polyvinylidene difluoride membranes (Millipore, Billerica, MA, USA). The membranes were then incubated with the primary antibodies in PBS containing 0.2\% Tween-20 (TBST) and 5\% bovine serum albumin overnight at $4^{\circ} \mathrm{C}$. After washing with TBST, the membranes were probed with a secondary antibody conjugated with horseradish peroxidase (Beijing Zhongshan Jinqiao Biological Technology Co., Ltd., Beijing, China) diluted 10,000-fold in PBS. Finally, an enhanced ECL chemiluminescence reagent kit (Millipore, Billerica, MA, USA) was used to detect the protein of interest in accordance with the manufacturer's protocol.

Statistical analysis

Statistical analyses were performed with GraphPad PRISM Version 6 (GraphPad Software, San Diego, CA, USA). All the results were expressed as the mean \pm standard error of the mean (SEM) of the data 
Fig. 1. ESAT-6 induces apoptosis in a time-dependent manner. (A) Macrophages were stimulated with $5 \mu \mathrm{g} / \mathrm{ml}$ ESAT-6 for various times, and the protein expression of caspase-3 in macrophages was assayed by western blotting. A representative blot of three experiments is shown. (B) Flow cytometric analysis of apoptosis of RAW264.7 cells treated with 5 $\mu \mathrm{g} / \mathrm{ml}$ ESAT-6. The flow cytometry plot shown is a representative of three experiments.

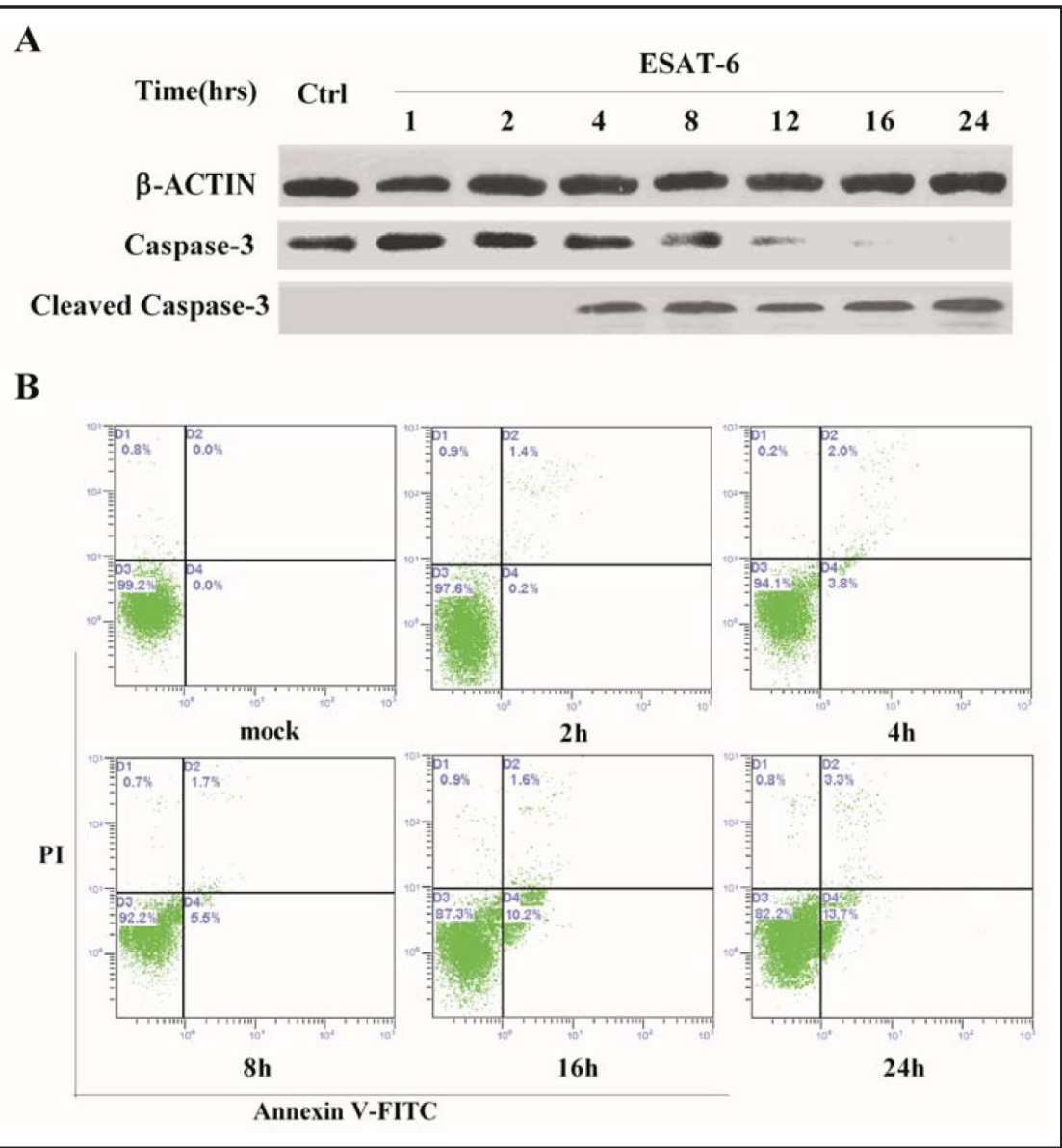

obtained from at least three experiments. Statistical analyses were performed using the unpaired two-tailed Student's t-test or one-way analysis of variance as appropriate. Comparison of nonparametric data between the groups was performed using the Mann-Whitney U, Kruskal Wallis, and Wilcoxon Rank tests. A p value of $<0.05$ was considered statistically significant.

\section{Results}

ESAT-6 induces apoptosis of RAW264.7 macrophages in a time-dependent manner

In order to demonstrate the effect of ESAT- 6 on the apoptosis of macrophages, we first analyzed the changes in caspase- 3 expression following ESAT- 6 treatment. There was a notable increase in cleaved caspase-3 in RAW264.7 cells (Fig. 1A) and a time-dependent increase in apoptotic cells (Fig. 1B).

miR-155 is required for ESAT-6-mediated macrophage apoptosis

As shown in Figure 2, our qPCR analyses indicated that there was a marked timedependent increase in miR-155 expression at $4 \mathrm{~h}$ after ESAT-6 treatment in RAW264.7 cells (Fig. 2A). Similar upregulation of the BIC transcript was also found in the present study (Fig. 2B).

We further examined the effect of miR-155 on ESAT-6-mediated macrophage apoptosis. After the RAW264.7 cells were treated with the lentivirus-mediated miRNA sponge for miR155 inhibition (Fig. 2C), a significant reduction of cleaved caspase-3 activity (Fig. 2D) and an obvious decrease in apoptotic populations (Fig. 2E) were observed in ESAT-6-treated macrophages, compared with the control cells. A similar reduction of cleaved caspase- 3 activity was also observed in BCG- or $M t b$-treated macrophages due to miR-155 inhibition 


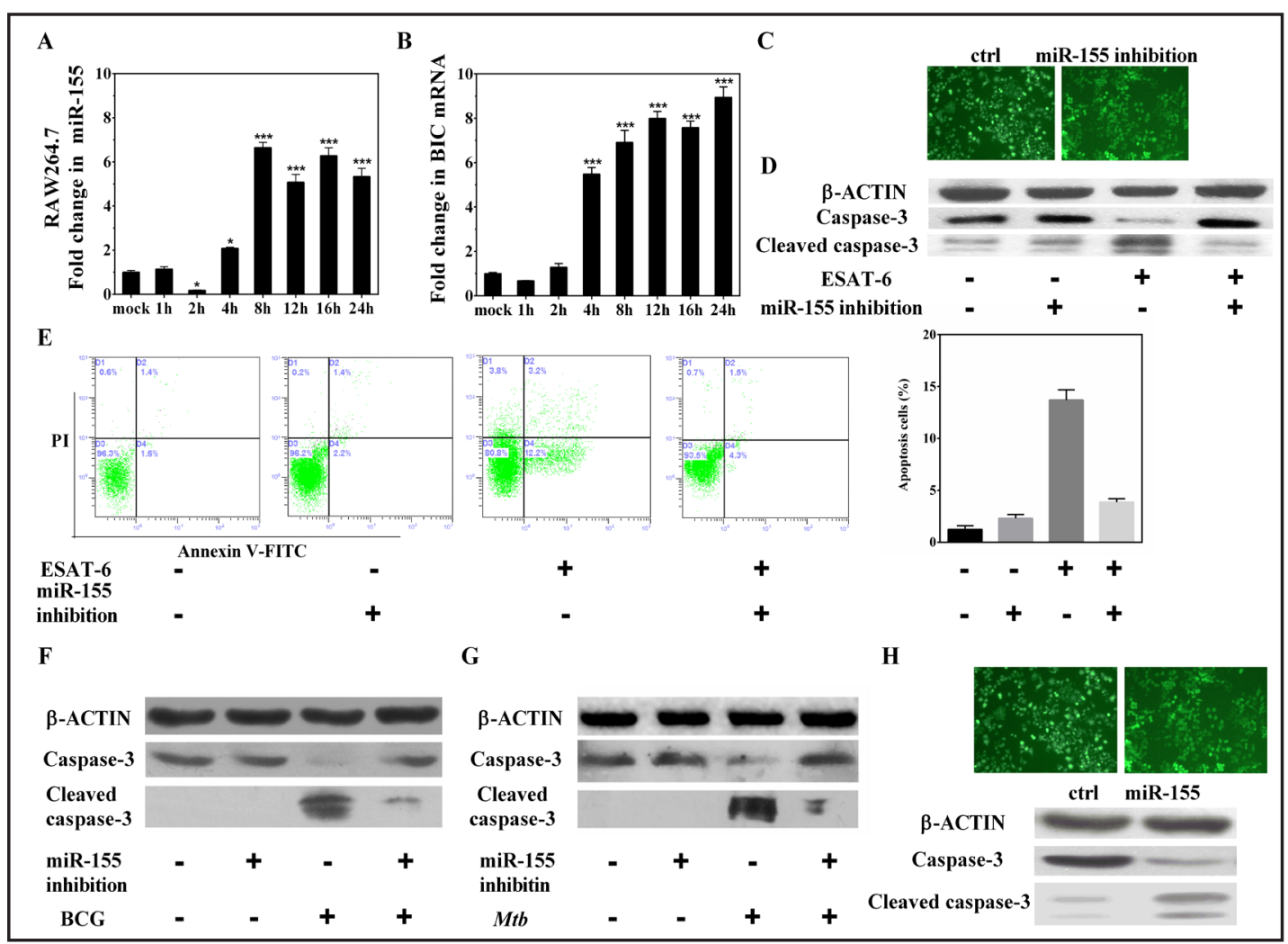

Fig. 2. Induced miR-155 expression is required for ESAT-6-mediated apoptosis in macrophages. The time courses of ESAT-6-induced miR-155 (A) and BIC transcript levels (B) as quantitated by qPCR. Total RNAs were harvested at different time points after ESAT- 6 treatment, and mRNA transcripts were determined by qPCR. MiR-155 was evaluated with the TaqMan microRNA assay kit using specific qRT primer sets, following the manufacturer's instructions. U6 snRNA was used for normalization. BIC gene expression was normalized to the internal control, $\beta$-actin. All values are expressed as means \pm SEM. Each experiment was performed in triplicate. '*' and '***' denote significant differences ( $\mathrm{p}<0.05$ and $\mathrm{p}<0.001$, respectively) as compared with the mock group. (C) RAW264.7 cells were transduced with lentiviral vectors expressing a scrambled control or miR-155 sponge with an MOI of approximately twenty. (D) The protein expression of caspase-3 was assayed by western blotting after the macrophages were treated with miR-155 inhibitor or controls. Macrophages were treated with $5 \mu \mathrm{g} / \mathrm{ml}$ ESAT- 6 or control for $24 \mathrm{~h}$, and caspase-3 protein levels were analyzed by western blots. (E) Flow cytometric analysis of apoptosis in RAW264.7 cells treated with ESAT- 6 or control for $24 \mathrm{~h}$. The flow cytometry plot shown is a representative of three experiments. Data are expressed as means \pm SEM, $n=3$. (F) The effect of miR-155 sponge on apoptosis during BCG infection. (G) The effect of miR-155 sponge on apoptosis during $M t b$ infection. The expression of caspase-3 was assayed in RAW264.7 cells under different treatments as indicated. (H) The effect of lentivirus-mediated miR-155 overexpression on the expression of caspase-3 in macrophages. RAW264.7 cells were transduced with lentiviral vectors expressing a scrambled control or miR-155 overexpression with MOIs of approximately 20 and 50, respectively. The expression of caspase- 3 was assayed by western blot. A representative blot of three experiments is shown.

(Fig. 2F and G). Furthermore, the overexpression of miR-155 could trigger the activation of apoptotic cascades in macrophages by the generation of active caspase-3 (Fig. 2H). Taken together, our results suggest that miR-155 plays a critical role in the induction of macrophage apoptosis.

ESAT-6 promotes macrophage apoptosis by targeting miR-155-SOCS1

Considering that SOCS1 is a proven cellular target of miR-155 in breast cancer [16], we determined whether ESAT-6-induced miR-155 expression could result in suppression KARGER 


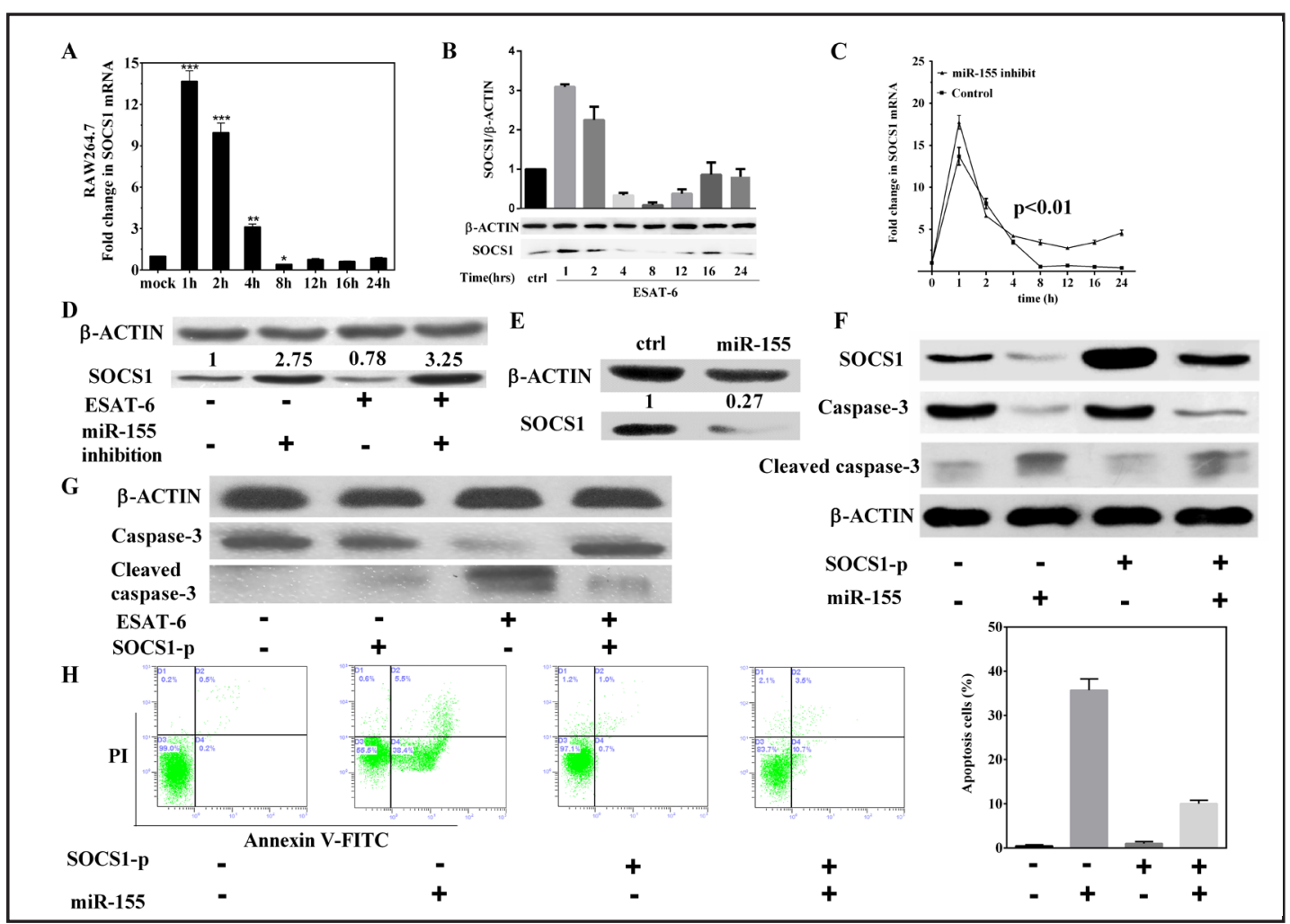

Fig. 3. ESAT-6 promotes macrophage apoptosis by targeting miR-155-SOCS1. (A) The time course of ESAT6-induced SOCS1 expression as quantitated by qPCR. SOCS1 gene expression was normalized to the internal control $\beta$-actin. All values are expressed as means \pm SEM. Each experiment was performed in triplicate. '*', ' $* *$ ', and ' $* * *$ ' denote significant differences $(\mathrm{p}<0.05, \mathrm{p}<0.01$, and $\mathrm{p}<0.001$, respectively) as compared with the mock group. (B) The time course of ESAT-6-induced SOCS1 expression as assayed by western blotting. The data are expressed as means \pm SEM $(n=3)$. (C) The time course of ESAT-6-induced SOCS1 expression in RAW264.7 cells infected with the scrambled control-expressing or miR-155 sponge-expressing lentivirus using real-time quantitative RT-PCR ( $\mathrm{n}=3$ ). (D) The expression of SOCS1 as assayed by western blotting at $24 \mathrm{~h}$ after treatment with the miR-155 inhibitor or control. The data are expressed as means \pm SEM $(n=3)$. (E) Overexpression of miR-155 downregulates SOCS1 in macrophages. The expression of SOCS1 protein was assayed by western blotting in macrophages after infection with the scrambled control-expressing or miR-155-overexpressing lentivirus. The data are expressed as means \pm SEM $(n=3)$. (F) The effect of miR155 on apoptosis under conditions of forced overexpression of SOCS1. The protein expression of caspase-3 was assayed by western blotting in RAW264.7 cells with different treatments as indicated. The figure shows a representative blot of three experiments with similar results. (G) The effect of lentiviral-mediated SOCS1 overexpression on caspase-3 expression in macrophages. The expression of caspase-3 was analyzed by western blotting. A representative blot of three experiments is shown. (H) The effect of miR-155 on apoptosis under conditions of forced overexpression of SOCS1 by using flow cytometry. The flow cytometry plot shown is a representative of three experiments. Data are expressed as means \pm SEM, $n=3$.

of SOCS1 expression. Our qPCR analyses (Fig. 3A) and western blotting results (Fig. 3B) demonstrated that SOCS1 was rapidly induced after ESAT-6 treatment and then was downregulated, most likely due to the time-dependent induction of miR-155 expression (Fig. 2A).

Additional qPCR analyses (Fig. 3C) and western blotting results (Fig. 3D) demonstrated that miR-155 inhibition led to a significant increase in the SOCS1 level in the presence of ESAT-6. Furthermore, overexpression of miR-155 could substantially inhibit the expression of SOCS1 (Fig. 3E), leading to a significant increase in cleaved caspase-3 activity (Fig. 3F) 


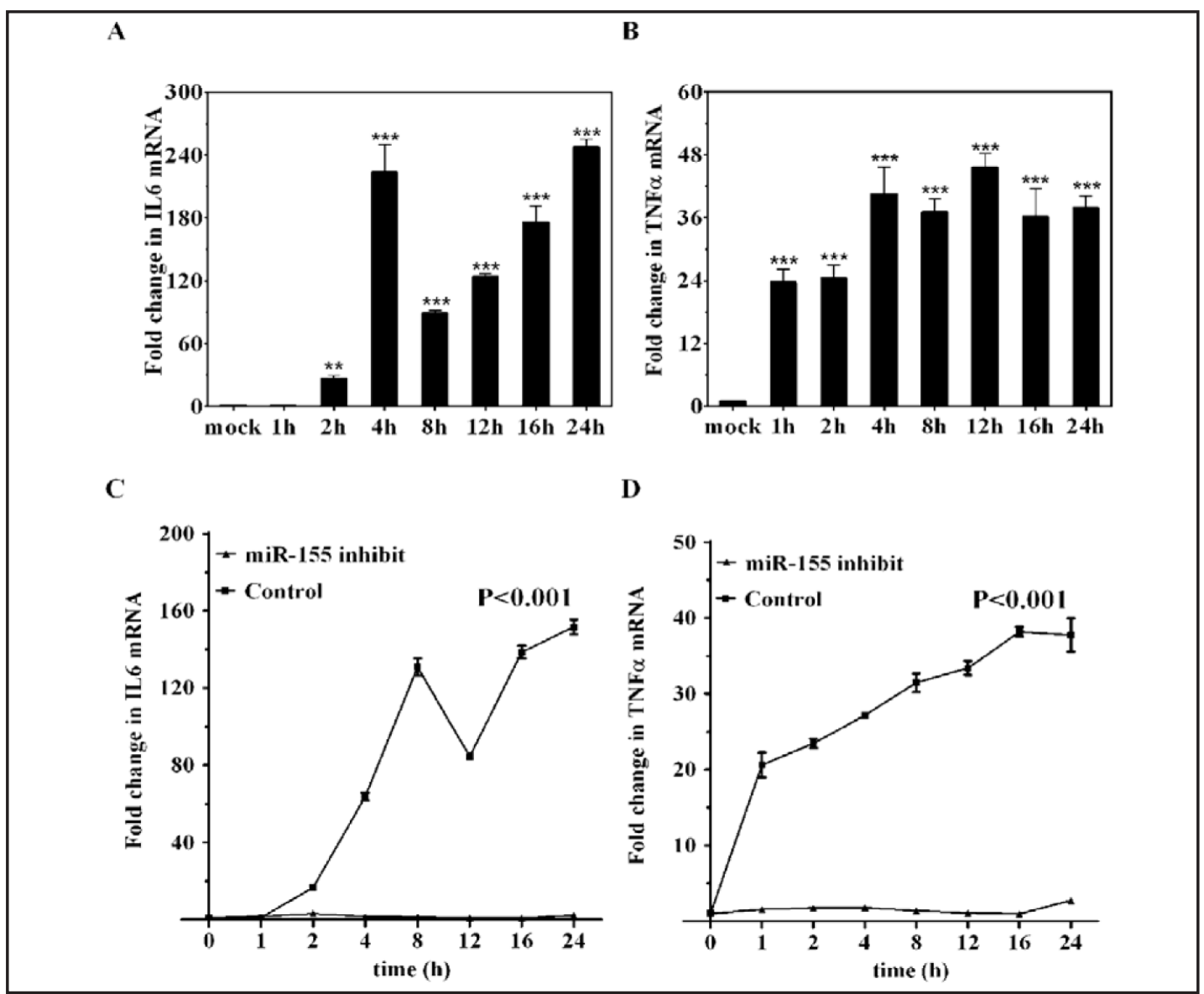

Fig. 4. ESAT- 6 enhances IL- 6 and TNF- $\alpha$ levels in macrophages. The time course of ESAT-6-induced IL-6 (A) and TNF- $\alpha$ (B) expression in macrophages as quantitated by qPCR. '**' and '***' denote significant differences ( $p<0.01$ and $p<0.001$, respectively) as compared with the mock group. The time course of ESAT-6-induced IL-6 (C) and TNF- $\alpha$ (D) expression after treatment with the miR-155 inhibitor or control. All values are expressed as means \pm SEM $(n=3)$.

and apoptotic cells under conditions of forced overexpression of SOCS1 (Fig. 3H). Moreover, overexpression of SOCS1 in RAW264.7 cells inhibited ESAT-6-mediated macrophage apoptosis by inactivation of caspase-3 activity (Fig. 3G). Taken together, these observations demonstrated that miR-155 targeted SOCS1, which had an important role in the ESAT-6mediated apoptosis of macrophages.

MiR-155 is essential for the ESAT-6-mediated protective immune response in macrophages

Next, we examined the expression of selected cytokines (IL-6, TNF- $\alpha$, IFN- $\gamma$, and iNOS) by qRT-PCR after ESAT- 6 treatment of RAW264.7 cells. Time-course studies showed that IL- 6 (Fig. 4A) and TNF- $\alpha$ (Fig. 4B) were substantially increased; while no changes in IFN- $\gamma$ or iNOS mRNA expression levels were observed (data not shown).

We also demonstrated that the expression levels of IL-6 (Fig. 4C) and TNF- $\alpha$ (Fig. 4D) were significantly decreased in macrophages treated with the miR-155 inhibitor, likely because of the induced expression of SOCS1 (Fig. 3C) in miR-155-inhibited macrophages. Taken together, these observations demonstrated that miR-155 was essential for the ESAT6-mediated protective immune response against $M t b$ in macrophages.

ESAT-6-induced expression of miR-155 is dependent on TLR2/NF- $\kappa B$ activation

Recent reports have suggested that ESAT- 6 can directly bind to TLR2 $[9,22]$ and that NF$\kappa \mathrm{B}$ activation is involved in the induction of BIC/miR-155 [23]. We postulated that ESAT-6- 


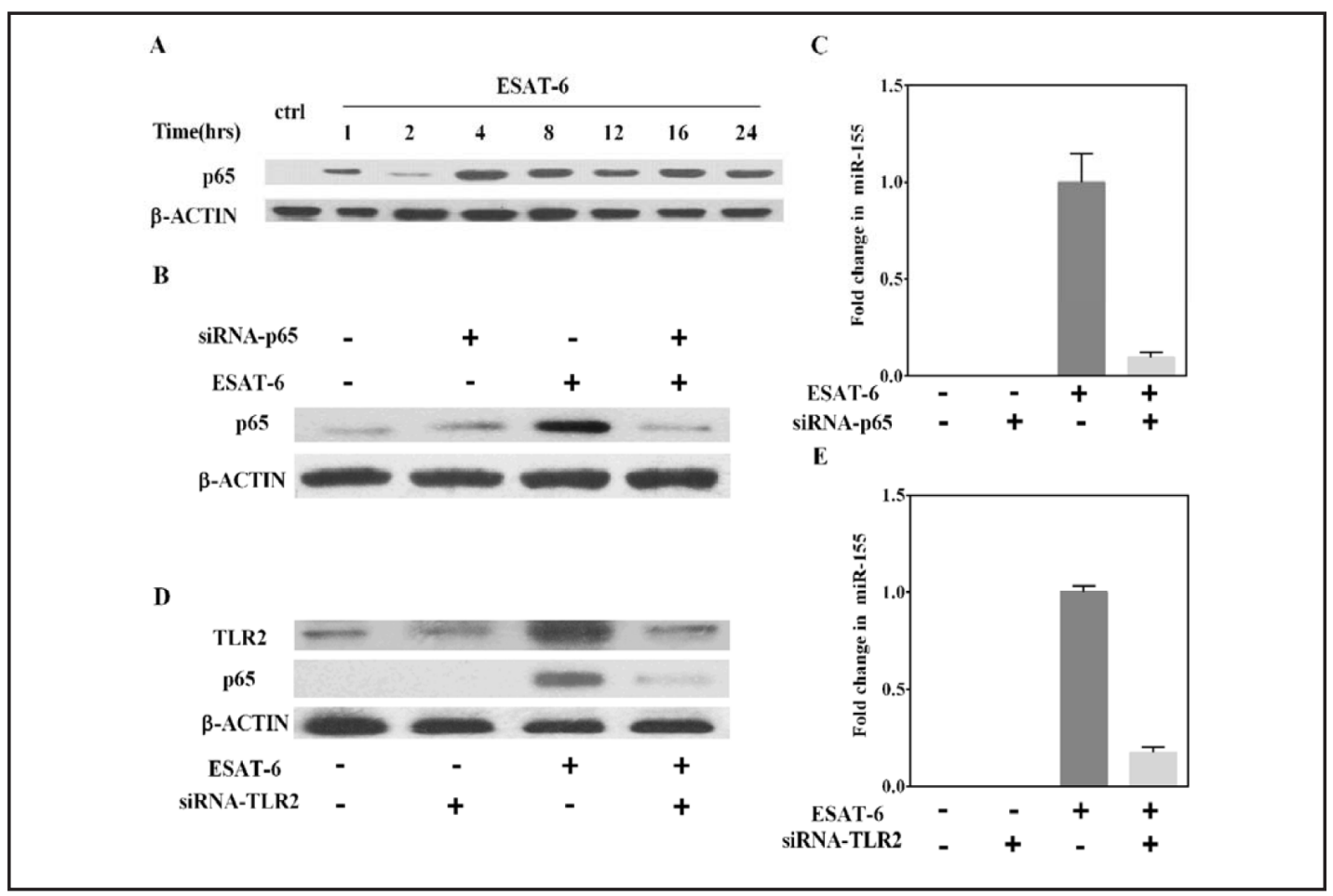

Fig. 5. ESAT-6-induced miR-155 expression is dependent on TLR2/NF- $\kappa B$ activation. (A) The time course of ESAT-6-induced p65 protein expression as assayed by western blotting. Macrophages were stimulated with $5 \mu \mathrm{g} / \mathrm{ml}$ ESAT- 6 and assayed for p65 protein levels using western blotting. A representative blot of three experiments is shown. The effect of p65-specific siRNA on p65 (B) and miR-155 (C) expression in RAW264.7 cells under different treatments as indicated. The effect of TLR2-specific siRNA on TLR2, p65 (D), and miR-155 (E) expression in RAW264.7 cells under different treatments as indicated. MiR-155 was evaluated with the TaqMan microRNA assay kit using specific qRT primer sets, following the manufacturer's instructions. U6 snRNA was used for normalization. All values are expressed as means \pm SEM. Each experiment was performed in triplicate.

induced miR-155 expression is TLR2-dependent. First, we determined the expression of p65 in RAW264.7 cells. As shown in Figure 5A, p65 expression was significantly upregulated at 4 $\mathrm{h}$ after ESAT-6 treatment. The small interfering RNA silencing of p65 (Fig. 5B) abrogated the ESAT-6-induced miR-155 expression (Fig. 5C), indicating a critical role of NF- $\kappa \mathrm{B}$ activation in the induction of miR-155.

Moreover, we also found that small interfering RNA silencing of TLR2 blocked ESAT-6induced NF- $\kappa$ B activation (Fig. 5D) and repealed ESAT-6-induced miR-155 expression (Fig. $5 \mathrm{E})$. These data indicate that the ESAT-6-induced expression of miR-155 is dependent on TLR2/NF- $\kappa$ B activation.

Differential expressions of TLR2, BIC, and SOCS1 in PBMCS of patients with active TB and LTB

TLR2, SOCS1, and BIC are key regulators of cytokine secretion and function. Previous studies have reported that the upregulation of SOCS1 is associated with suppression of immune activation of $\mathrm{T}$ cells, favoring the survival of $M t b$ [24]. In the present study, we examined the expression levels of TLR2, BIC, and SOCS1 in human PBMCs of patients with active TB disease and LTB disease, respectively. Interestingly, both TLR2 and BIC mRNA expression levels were increased in the patients with LTB, compared to their counterparts with TB $(\mathrm{p}<0.01)$ and the healthy non-TB subjects $(\mathrm{p}<0.01)$. In addition, the BIC mRNA transcripts were decreased in the TB group, compared with the control group $(p<0.05)$; while the TLR2 mRNA levels were similar in both the TB and control groups (Fig. 6A-B). 


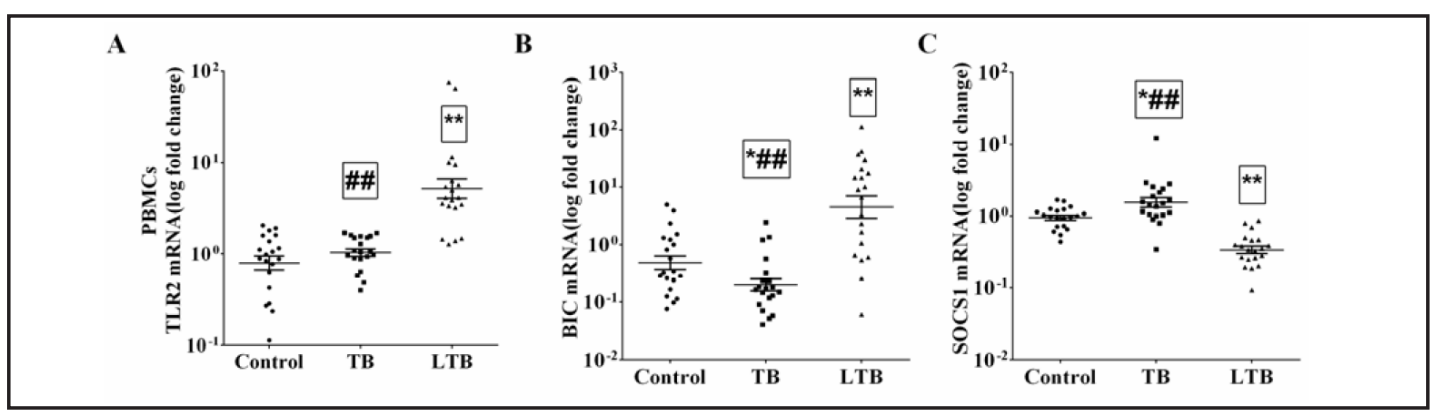

Fig. 6. Expression profiles of TLR2, BIC, and SOCS1 in PBMCs of patients with active TB or latent TB. PBMCs $\left(10^{6}\right)$ from volunteers with TB negative (Control, $n=20$ ), active TB (TB, $n=20$ ), or latent TB (LTB, $n=20$ ). Total RNA was harvested, and mRNA transcripts were determined by qPCR. The gene expression levels of TLR2, BIC, and SOCS1 were normalized to the internal control GAPDH. The data are represented by a scatter plot with the median values of each group indicated by a horizontal line. '*' and '**' denote significant differences ( $\mathrm{p}<0.05$ and $\mathrm{p}<0.01$, respectively) as compared with the control; while '\#\#' denotes significant differences ( $p<0.01)$ as compared with the LTB group using the Mann-Whitney U test. (A) TLR2; (B) BIC; and (C) SOCS1 mRNA expression.

Fig. 7. Proposed mechanism of action by which ESAT-6/TLR2 signaling induces miR-155. The signaling network may be involved in the suppression of the negative feedback inhibition of TLR2-mediated protective immune response and induction of macrophage apoptosis against mycobacterial infections.

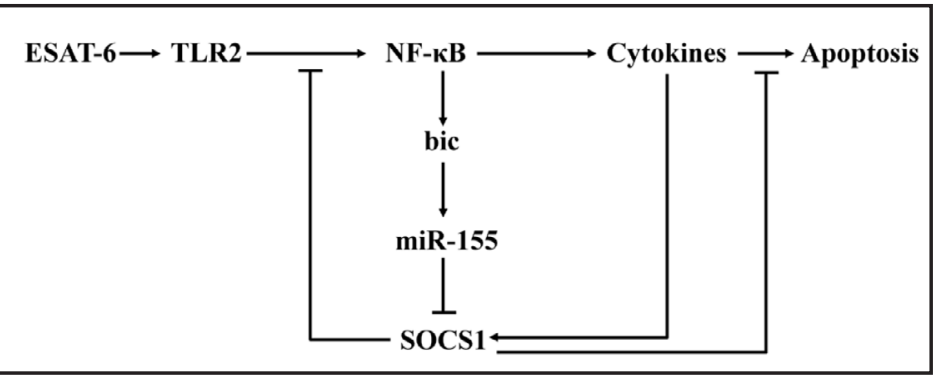

Additionally, the SOCS1 mRNA expression levels were found to be decreased in the LTB group, compared with the TB $(\mathrm{p}<0.01)$ and control groups ( $<0.01)$; whereas the same levels were much higher in the TB group than in the control group $(\mathrm{p}<0.05$; Fig. $6 \mathrm{C})$. Collectively, these findings indicate that an increase of TLR2 and BIC expression levels in LTB may be involved in the regulation of host immunity to $M t b$.

\section{Discussion}

A previous study [17] has demonstrated that miR-155 is highly expressed in ESAT-6treated macrophages, but the role of miR-155 in ESAT-6-induced apoptosis of macrophages is not fully understood. In the present study, we showed for the first time that the TLR2/ miR-155/SOCS1 signaling axis had an important role in the ESAT-6-mediated protective immune response and macrophage apoptosis. Specifically, miR-155 was induced upon ESAT6 stimulation that was dependent on TLR2/NF- $\kappa B$ activation, thus promoting apoptosis by targeting the miR-155-SOCS1 interaction. We further showed that pro-inflammatory mediators including IL- 6 and TNF- $\alpha$ were increased in macrophages after ESAT- 6 treatment, likely because of the downregulation of SOCS1 by miR-155. We believe this is a novel mechanism by which ESAT-6 mediates a protective immune response and macrophage apoptosis (Fig. 7).

Currently, there are three main approaches used in miRNA loss-of-function studies: gene knockout, chemically modified antisense oligonucleotides, and miRNA sponges. More recently, lentivirus-mediated expression of miRNAs and miRNA sponges have been used to 
generate and isolate stable gain- and loss-of-function phenotypes for individual miRNAs in a wide range of target cells, thanks to the stable and high transfection efficacy [25-27]. In the present study, the importance of miR-155 in ESAT- 6 regulation of innate immunity and apoptosis was investigated using a lentivirus-mediated miR-155 sponge. The lentiviral vector encoding the miR-155 sponge was found to sufficiently silence miR-155, resulting in the upregulation of SOCS1, which could attenuate the production of cytokines (such as IL-6 and TNF- $\alpha$ ) and ultimately suppress the immune response to $M t b$. Moreover, miR-155 inhibition could suppress macrophage apoptosis, while lentivirus-mediated miR-155 overexpression caused induction of apoptosis.

Considering that miR-155 transcription is dependent on NF- $\kappa B$ activation [23], we further investigated the expression of p65 in RAW264.7 cells upon ESAT-6 stimulation. It appeared contradictory that NF- $\mathrm{KB}$ activation was induced at $4 \mathrm{~h}$ in macrophages, while TLR/ MyD88-dependent NF- $\kappa$ B activation could be inhibited within $1 \mathrm{~h}$ after exogenous ESAT- 6 pretreatment [22]. However, it should be noted that NF- $\mathrm{BB}$ activation could be modulated by ESAT- 6 with a temporal and spatial specificity. Moreover, miR-155 induction may be dependent on several other transcription factors such as ETS2 [12], AP-1 [28], and SMAD4 [29]. Therefore, further investigations are necessary to determine the other mechanisms responsible for the regulation of miR-155 in macrophages.

Most individuals infected with $M t b$ are asymptomatic, known as LTB, which may persist throughout a lifetime, indicating that immune responses are closely associated with the outcome of TB. TLR2, SOCS1, and BIC serve as key regulators of immune responses and contribute to the regulation of immune activation of $\mathrm{T}$ cells and macrophages after infection with $M t b$. In the present study, we found that the TLR2, BIC, and SOCS1 mRNA expression levels were differentially expressed in human PBMCs of patients with TB and LTB, respectively. The upregulation of TLR2 and BIC in LTB patients may contribute to host immunity against $M t b$, while increased expression of SOCS1 may be associated with the suppression of immune activation, favoring the survival of $M t b$ in TB patients. Furthermore, our results indicate that TLR2, BIC, and SOCS1 could be potential biomarkers for discriminating LTB infection and TB disease.

Besides SOCS1, miR-155 also has other targets involved in immune functions, such as SHIP1 [30] and C/EBP $\beta$ [31], two important negative regulators of the IL-6 signaling pathway. Thus, it is speculated that ESAT- 6 may modulate immune response by regulating SHIP-1, C/ EBP $\beta$, or both, via miR-155. This speculation remains to be investigated in future studies.

In summary, the results presented in this study demonstrated that ESAT- 6 promoted apoptosis in macrophages by targeting the miR-155-SOCS1 interaction. We concluded that miR-155 also had a critical role in EAST-6-mediated protective immune responses against $M t b$. Furthermore, the enhanced expression of miR-155 was largely dependent on TLR2/ NF- $\kappa B$ activation. In addition, our study suggested that differential expression levels of TLR2, BIC, and SOCS1 could be involved in regulating the protective immune response against $M t b$ infection. Overall, the TLR2/miR-155/SOCS1 signaling axis regulates ESAT-6-mediated host immunity and apoptosis in macrophages, which might serve as a novel target for immunotherapy for $M t b$ infection. Therefore, this study provides a basis for a new avenue to explore various immunomodulatory mechanisms of ESAT-6 in host innate immunity against mycobacterial infections.

\section{Acknowledgements}

This work was supported by the National Natural Science Foundation of China (81371760) and the National Infection Disease Prevention and Cure Special Project of China (2013ZX10003006-003-002).

\section{Disclosure Statement}

The authors declare no competing interests.

\section{KARGER}




\section{References}

1 Zumla A, George A, Sharma V, Herbert N, Baroness Masham of I: WHO's 2013 global report on tuberculosis: successes, threats, and opportunities. Lancet 2013;382:1765-1767.

2 Chen M, Gan H, Remold HG: A mechanism of virulence: virulent Mycobacterium tuberculosis strain H37Rv, but not attenuated H37Ra, causes significant mitochondrial inner membrane disruption in macrophages leading to necrosis. J Immunol 2006;176:3707-3716.

3 Behar SM, Martin CJ, Booty MG, Nishimura T, Zhao X, Gan HX, Divangahi M, Remold HG: Apoptosis is an innate defense function of macrophages against Mycobacterium tuberculosis. Mucosal Immunol 2011;4:279-287.

4 Nishimura T, Zhao X, Gan H, Koyasu S, Remold HG: The prostaglandin E2 receptor EP4 is integral to a positive feedback loop for prostaglandin E2 production in human macrophages infected with Mycobacterium tuberculosis. FASEB J 2013;27:3827-3836.

5 Bai Y, Xue Y, Gao H, Wang L, Ding T, Bai W, Fan A, Zhang J, An Q, Xu Z: Expression and purification of Mycobacterium tuberculosis ESAT-6 and MPT64 fusion protein and its immunoprophylactic potential in mouse model. Protein Expr Purif 2008;59:189-196.

6 Li W, Deng G, Li M, Zeng J, Zhao L, Liu X, Wang Y: A recombinant adenovirus expressing CFP10, ESAT6, Ag85A and Ag85B of Mycobacterium tuberculosis elicits strong antigen-specific immune responses in mice. Mol Immunol 2014;62:86-95.

7 Pym AS, Brodin P, Majlessi L, Brosch R, Demangel C, Williams A, Griffiths KE, Marchal G, Leclerc C, Cole ST: Recombinant BCG exporting ESAT- 6 confers enhanced protection against tuberculosis. Nat Med 2003;9:533-539.

8 Derrick SC, Morris SL: The ESAT6 protein of Mycobacterium tuberculosis induces apoptosis of macrophages by activating caspase expression. Cell Microbiol 2007;9:1547-1555.

9 Chatterjee S, Dwivedi VP, Singh Y, Siddiqui I, Sharma P, Van Kaer L, Chattopadhyay D, Das G: Early Secreted Antigen ESAT-6 of Mycobacterium tuberculosis Promotes Protective T Helper 17 Cell Responses in a TollLike Receptor-2-dependent Manner. Plos Pathogens 2011;7.

10 Mir SA, Verma I, Sharma S: Immunotherapeutic potential of recombinant ESAT-6 protein in mouse model of experimental tuberculosis. Immunol Lett 2014;158:88-94.

11 Murugaiyan G, Beynon V, Mittal A, Joller N, Weiner HL: Silencing microRNA-155 ameliorates experimental autoimmune encephalomyelitis. J Immunol 2011;187:2213-2221.

12 Quinn SR, Mangan NE, Caffrey BE, Gantier MP, Williams BR, Hertzog PJ, McCoy CE, O'Neill LA: The role of Ets2 transcription factor in the induction of microRNA-155 (miR-155) by lipopolysaccharide and its targeting by interleukin-10. J Biol Chem 2014;289:4316-4325.

13 Dominguez-Soto A, Puig-Kroger A, Vega MA, Corbi AL: PU.1 regulates the tissue-specific expression of dendritic cell-specific intercellular adhesion molecule (ICAM)-3-grabbing nonintegrin. J Biol Chem 2005;280:33123-33131.

14 Naka T, Narazaki M, Hirata M, Matsumoto T, Minamoto S, Aono A, Nishimoto N, Kajita T, Taga T, Yoshizaki K, Akira S, Kishimoto T: Structure and function of a new STAT-induced STAT inhibitor. Nature 1997;387:924929.

15 Fujimoto M, Naka T: Regulation of cytokine signaling by SOCS family molecules. Trends Immunol 2003;24:659-666.

16 Jiang S, Zhang HW, Lu MH, He XH, Li Y, Gu H, Liu MF, Wang ED: MicroRNA-155 functions as an OncomiR in breast cancer by targeting the suppressor of cytokine signaling 1 gene. Cancer Res 2010;70:3119-3127.

17 Kumar R, Halder P, Sahu SK, Kumar M, Kumari M, Jana K, Ghosh Z, Sharma P, Kundu M, Basu J: Identification of a novel role of ESAT-6-dependent miR-155 induction during infection of macrophages with Mycobacterium tuberculosis. Cell Microbiol 2012;14:1620-1631.

18 Berry MP, Graham CM, McNab FW, Xu Z, Bloch SA, Oni T, Wilkinson KA, Banchereau R, Skinner J, Wilkinson RJ, Quinn C, Blankenship D, Dhawan R, Cush JJ, Mejias A, Ramilo O, Kon OM, Pascual V, Banchereau J, Chaussabel D, O'Garra A: An interferon-inducible neutrophil-driven blood transcriptional signature in human tuberculosis. Nature 2010;466:973-977. 
Yang et al.: ESAT-6, MIR-155/SOCS1 and Macrophage Apoptosis

19 Frahm M, Goswami ND, Owzar K, Hecker E, Mosher A, Cadogan E, Nahid P, Ferrari G, Stout JE: Discriminating between latent and active tuberculosis with multiple biomarker responses. Tuberculosis (Edinb) 2011;91:250-256.

20 Dhanasekaran S, Jenum S, Stavrum R, Ritz C, Faurholt-Jepsen D, Kenneth J, Vaz M, Grewal HM, Doherty TM, Group TBTS: Identification of biomarkers for Mycobacterium tuberculosis infection and disease in BCGvaccinated young children in Southern India. Genes Immun 2013;14:356-364.

21 Abi Abdallah DS, Egan CE, Butcher BA, Denkers EY: Mouse neutrophils are professional antigen-presenting cells programmed to instruct Th1 and Th17 T-cell differentiation. Int Immunol 2011;23:317-326.

22 Pathak SK, Basu S, Basu KK, Banerjee A, Pathak S, Bhattacharyya A, Kaisho T, Kundu M, Basu J: Direct extracellular interaction between the early secreted antigen ESAT- 6 of Mycobacterium tuberculosis and TLR2 inhibits TLR signaling in macrophages. Nat Immunol 2007;8:610-618.

23 Gatto G, Rossi A, Rossi D, Kroening S, Bonatti S, Mallardo M: Epstein-Barr virus latent membrane protein 1 trans-activates miR-155 transcription through the NF-kappaB pathway. Nucleic Acids Res 2008;36:66086619.

24 Almeida AS, Lago PM, Boechat N, Huard RC, Lazzarini LC, Santos AR, Nociari M, Zhu H, Perez-Sweeney BM, Bang H, Ni Q Huang J, Gibson AL, Flores VC, Pecanha LR, Kritski AL, Lapa e Silva JR, Ho JL: Tuberculosis is associated with a down-modulatory lung immune response that impairs Th1-type immunity. J Immunol 2009;183:718-731.

25 Ebert MS, Neilson JR, Sharp PA: MicroRNA sponges: competitive inhibitors of small RNAs in mammalian cells. Nat Methods 2007;4:721-726.

26 Scherr M, Venturini L, Battmer K, Schaller-Schoenitz M, Schaefer D, Dallmann I, Ganser A, Eder M: Lentivirus-mediated antagomir expression for specific inhibition of miRNA function. Nucleic Acids Res 2007;35:e149.

27 Chen L, Zhang K, Shi Z, Zhang A, Jia Z, Wang G, Pu P, Kang C, Han L: A lentivirus-mediated miR-23b sponge diminishes the malignant phenotype of glioma cells in vitro and in vivo. Oncol Rep 2014;31:1573-1580.

28 Yin Q, Wang X, McBride J, Fewell C, Flemington E: B-cell receptor activation induces BIC/miR-155 expression through a conserved AP-1 element. J Biol Chem 2008;283:2654-2662.

29 Kong W, Yang H, He L, Zhao JJ, Coppola D, Dalton WS, Cheng JQ: MicroRNA-155 is regulated by the transforming growth factor beta/Smad pathway and contributes to epithelial cell plasticity by targeting RhoA. Mol Cell Biol 2008;28:6773-6784.

30 O'Connell RM, Chaudhuri AA, Rao DS, Baltimore D: Inositol phosphatase SHIP1 is a primary target of miR155. Proc Natl Acad Sci U S A 2009;106:7113-7118.

31 Costinean S, Sandhu SK, Pedersen IM, Tili E, Trotta R, Perrotti D, Ciarlariello D, Neviani P, Harb J, Kauffman LR, Shidham A, Croce CM: Src homology 2 domain-containing inositol-5-phosphatase and CCAAT enhancer-binding protein beta are targeted by miR-155 in B cells of Emicro-MiR-155 transgenic mice. Blood 2009;114:1374-1382. 